Voix et Images

volxetimages

\title{
Naïm Kattan ou la fortune du migrant
}

Jacques Allard

Volume 11, numéro 1, automne 1985

\section{Naïm Kattan}

URI : https://id.erudit.org/iderudit/200533ar

DOI : https://doi.org/10.7202/200533ar

Aller au sommaire du numéro

Éditeur(s)

Université du Québec à Montréal

ISSN

0318-9201 (imprimé)

1705-933X (numérique)

Découvrir la revue

Citer cet article

Allard, J. (1985). Naïm Kattan ou la fortune du migrant. Voix et Images, 11(1),

7-9. https://doi.org/10.7202/200533ar d'utilisation que vous pouvez consulter en ligne.

https://apropos.erudit.org/fr/usagers/politique-dutilisation/ 


\title{
Naïm Kattan \\ ou la fortune du migrant
}

\author{
par Jacques Allard, Université du Québec à Montréal
}

Natif de Bagdad, N. Kattan est au Québec depuis 1954, après avoir vécu l'après-guerre à Paris. Il a participé à la préparation et à l'éclosion de notre "révolution tranquille», se faisant dès le début un communicateur multimédiatique, chroniqueur littéraire aussi bien que journaliste de politique étrangère, patient prospecteur de l'interculture québéco-canadienne. Il fut à Montréal ce qu'il était déjà à Paris ou plus tôt à Bagdad: un voyageur du transculturel, soucieux de comprendre les rapports de l'Orient et de l'Occident et tout aussi bien ceux des groupes ethniques canadiens. Juif d'Arabie, Arabe de la judéité, oriental d'Occident, occidental d'Orient, l'homme de Bagdad est inépinglable; ce francophone québécois est toujours ailleurs que là où on le fixe quand on ne veut pas comprendre la richesse du désert sémitique originel. Et son discours: celui du migrant, fatalement. D'où le titre de notre dossier, inspiré d'ailleurs de celui de son prochain roman: la Fortune du passager.

L'écrivant hyper actif, devenu administrateur culturel, fondateur (en 1967) du service des Lettres au Conseil des arts du Canada, devait finalement se manifester dans son ambition première et dernière: être écrivain. D'abord essayiste, il fut et reste encore dramaturge, tout en étant de plus en plus nouvelliste et romancier. La qualité de son travail dans tout cet espace social et textuel devait lui valoir, au fil du temps, une notoriété certaine dont témoigne, par exemple, le prix France-Canada (décerné en 1971) pour l'essai le Réel et le théâtral.

Voix et images a cru venu le moment de rencontrer l'écrivain et de faire le point sur l'ensemble de ses activités. Tout en voulant marquer le trentième anniversaire d'une vie littéraire québécoise, il s'agissait de faire avancer la réflexion sur une œuvre qui reste étonnamment peu étudiée, en dépit de l'importante couverture de presse dont elle a fait l'objet. En ces temps postréférendaires (interminables) où les Québécois PLV (pure laine vierge) interrogent comme un miroir la néo-québécité (surtout celle des autres minorités ethno-linguistiques qui les entourent) il devrait être utile de revenir à l'un d'entre nous (non PLV, mais tout de même francophone...). Son discours de migrant nous dit et nous fait sans doute davantage que toutes les propositions érabliérantes et coniférantes, à condition de se souvenir de quelques racines amérindiennes et autres souches de sangs-mêlés.

Eût-il paru plus tôt, ce dossier aurait pu être plus étoffé. Ironie du sort: il ne put se réaliser qu'au moment où notre subventionneur québécois (le FCAR) diminuait sa subvention... Nous aurions aimé dépasser encore ici notre format habituel et publier un ensemble plus fourni. Ainsi, la bibliographie déjà fort respectable de Lucie Robert n'aura pu être augmentée des nouveaux titres traités par Chantal de Grandpré. Et l'étude de Sylvain Simard restera orpheline. Quant à l'entrevue, on en retrouvera plus bas une 
partie substantielle mais limitée à la facette romancière de l'œuvre. Il va de soi que la totalité documentaire reste à la disposition des chercheurs intéressés à nos archives. Mais il nous a semblé utile de signaler à nos lecteurs l'essentiel de la part non publiée.

\section{Sur les rapports à la langue et à la culture française:}

N. Kattan a précisé que le choix de la langue française pour un parlant arabe comportait ses difficultés propres. Qu'il lui a fallu ce long temps d'apprentissage alors qu'il s'est confiné à la pratique journalistique, où la communication est plus facile à établir puisque l'on n'écrit pas "de l'intérieur" de la langue. Par ailleurs, il a renoncé à certaine "pente" arabe, à cette propension à l'embellissement, au fleuri, comme à la tentation de faire carrière dans l'orientalisme à la française, si rentable pour certains contemporains. Autre donnée importante pour un oriental, les temps (passé, présent et futur) ont d'autres valeurs qu'en français, ce que tente de faire passer notre auteur dans son écriture. Il avoue aussi qu'il n'oublie pas facilement que, pour lui dans la langue parlée et écrite de son enfance, le soleil est féminin et la lune masculine.

\section{Sur l'écriture:}

Dans la préparation de ses essais, il rejette la grille ou le plan cartésien. Il préfère produire un premier texte, générateur, qu'il publiera ensuite en guise d'introduction. Puis le livre se développe par cercles concentriques, dans une sorte de structure spiralée dont on ne sort pas; au bout de laquelle on n'aboutit nulle -part, sinon à la reprise, au recommencement: c'est la forme que je donne à ma liberté, ne pas aboutir, sinon à un autre livre. Il définit son écriture comme moyen-orientale, coranique ou biblique.

\section{Sur les essais :}

Le Réel et le théâtral (1970) s'est ainsi écrit sous la forme initiale d'un article envoyé à la Nouvelle revue française. Il a dû le développer sur les instances de Jean Grosjean, poète et traducteur de la Bible, le produisant en correspondance et amitié avec celui qui allait aussi préfacer l'ouvrage édité par Maurice Nadeau aux "Lettres nouvelles» (chez Denoël). Le livre qui devait lui valoir deux rencontres avec André Malraux (il y voyait une "Tentation de l'Orient») continue de cheminer, suscitant de fréquentes réactions personnelles. Il est même question qu'il reparaisse en poche. Notre invité a par ailleurs insisté sur l'idée centrale du troisième essai, le Désir et le pouvoir (1983), à savoir qu'il n'est pas d'espace politique qui suffise au désir; que seul l'espace artistique peut le combler, ou encore, parce que plus accessible, l'espace religieux: d'où le retour de notre auteur aux trois grandes religions (judaïsme, christianisme et islamisme). J'admire tout ce qui est religieux, dit-il en précisant que pour lui la religion n'est pas affaire de système mais d'éthique; et qu'être juif est une manière d'être au monde, Israël n'étant d'ailleurs pas une «puissance», mais une «vision».

\section{Sur le théâtre:}

Dans ses pièces, il n'essaie pas de théâtraliser le réel, comme on l'a tant fait en Occident. Il veut plutôt, allant au-delà de l'échange de paroles, pratiquer un art du second degré qui fasse en sorte que le langage quotidien 
et banal utilisé en cache un autre, suggéré, qu'il s'agit de faire éclater sans violence. Comme dans l'Indiscrétion (publiée dans l'Avant-scène).

\section{Ses lectures:}

Il lit d'abord et tout le temps la Bible et Shakespeare. Du côté français, un ensemble d'écrivains. Cités: Racine, Molière, Chateaubriand, Baudelaire,.. puis il ajoute Proust qu'il relit actuellement et enfin Malraux auquel il prévoit revenir. Aux États-Unis, un seul nom: Saül Bellow:

\section{Sur la littérature québécoise:}

Là se trouve une humanité où(il) se reconnaît. Ses écrivains préférés? Des classiques: A. Hébert, G. Roy, A. Langevin, J. Brault, M.-C. Blais et G. Miron. Sur notre destin littéraire: il faut, dit-il affirmer notre différence ne pas se perdre dans une autre littérature (française). Etre un bon écrivain français pour être bien lu en France, c'est aussi dangereux que d'écrire en mauvais français. L'on doit, selon lui, éviter tout aussi bien le formalisme littéraire (qui correspond à un bon parler) que le joual. L'écriture est une convention entre l'écrivain et ses lecteurs; l'on doit, en conséquence, dépasser la parole brute mais tout autant l'insignifiante... pour se situer entre les deux pôles. Et puis, s'il a choisi de vivre au Québec c'est pour être vraiment dans un rapport d'échange (critique) avec la mère-culture française.

\section{Sur le Conseil des arts:}

N. Kattan tient à distinguer ses goûts littéraires de son devoir professionnel d'administrateur culturel. Il insiste sur le pouvoir absolu des jurys, une fois qu'ils sont constitués selon les règles strictes du Conseil. Même quand la direction doit honorer un séparatiste (ou autre taré, politiquement ou moralement parlant), sachant bien les réactions négatives à venir du Parlement ou d'ailleurs, elle défend le choix esthétique fait par les personnes compétentes réunies. Sur la question plus générale, qui revient souvent: pourquoi subventionner la culture? notre invité répond que tous les pays le font, d'une manière ou d'une autre (par l'État ou les fondations privées); parce qu'il s'agit d'un service public, pour la qualité de vie que cela donne. Et qu'autrement la souveraineté culturelle serait menacée. Exemple: faut-il ici dépendre des éditeurs étrangers (français et anglais) pour décider des auteurs (même autochtones) que l'on veut lire? C'est ainsi que la question doit être posée. Quant aux candidats aux subventions, ils sont de trois types: les amateurs enthousiastes qui ignorent ce qu'est une œuvre; les artistes frustrésenvieux; et les artistes qui croient aux valeurs d'échange, à la communauté artistique. N. Kattan insiste aussi pour signaler que son cuvre personnelle est écartée de tous les programmes administrés, même ceux qui veillent à l'achat de livres canadiens. De la même manière, il n'écrit ou n'intervient jamais dans les organes subventionnés sauf dans les publications auxquelles il collaborait avant son entrée en fonction (Liberté et les Écrits du Canada français). Et il n'a pu accepter cette entrevue que parce que Voix et images n'est pas soutenue financièrement par le Conseil des arts du Canada. Le même raisonnement vaut pour l'édition de ses livres. Ce puritanisme assure, dit-il, sa liberté d'écrivain.

Ainsi vont le discours et la fortune du migrant: de ville en ville, de pays en pays, de la vie à ses fictions. 